\title{
The observation likelihood of silence: analysis and prospects for VAD applications
}

\author{
Igor Odriozola ${ }^{1}$, Inma Hernaez ${ }^{1}$, Eva Navas ${ }^{1}$, Luis Serrano ${ }^{1}$, Jon Sanchez ${ }^{1}$ \\ ${ }^{1}$ AHOLAB, University of the Basque Country UPV/EHU \\ \{igor, inma, eva, lserrano, ion\}eaholab.ehu.eus
}

\begin{abstract}
This paper shows a research on the behaviour of the observation likelihoods generated by the central state of a silence HMM (Hidden Markov Model) trained for Automatic Speech Recognition (ASR) using cepstral mean and variance normalization (CMVN). We have seen that observation likelihood shows a stable behaviour under different recording conditions, and this characteristic can be used to discriminate between speech and silence frames. We present several experiments which prove that the mere use of a decision threshold produces robust results for very different recording channels and noise conditions. The results have also been compared with those obtained by two standard VAD systems, showing promising prospects. All in all, observation likelihood scores could be useful as the basis for the development of future VAD systems, with further research and analysis to refine the results.
\end{abstract}

Index Terms: VAD, observation likelihood, cepstral normalization

\section{Introduction}

Voice activity detection (VAD) is an important issue in Automatic Speech Recognition (ASR) or ASR-based systems. It allows the systems to reduce the computation cost and, as a consequence, the response time of the decoding process, by only passing speech frames [1]. If the access to the system is intended to be universal, the VAD has to cope with different noise levels, with no - or little- loss in accuracy. Indeed, the greatest challenge for the current ASR systems is to cope with background noise in the input speech signal [2].

A large number of speech features and combinations have been proposed for VAD [3]. Gaussian Mixture Models (GMM) and Hidden Markov Models (HMMs) have been tested in this context [4][5]. Recently, the use of classifiers has been very common: decision trees (DT) [6], Support Vector Machines (SVM) [7] and hybrid SVM/HMM architectures [8]. More recently, neural networks (NN) have appeared in the literature outperforming the previous designs [9][10][11]. However, these approaches are complex and do not work in real time.

Little research has been done using cepstral normalization for VAD proposals, although it proved to be rather discriminative already in [12]. Here, we introduce some research on the use of observation likelihoods for VAD, applying Cepstral Mean and Variance Normalization (CMVN). We analyse the behaviour of the observation likelihoods generated by the GMM in the central state of the silence HMMs trained for ASR. Results show that it is a promising basis for future prospects.

The next section is a study of different aspects of the observation likelihood scores. Section 3 describes the databases and metrics used for the experiments. Then, VAD some experiments are shown in section 4. Finally, some conclusions and future prospects are explained in section 5 .

\section{The observation likelihood}

In speech recognition, audio segments corresponding to the same recognition unit (word, phone, triphone etc., even silence or non-speech) are gathered and processed, in order to extract acoustic parameters from them - typically Mel-frequency cepstral coefficients (MFCC) — and train a different acoustic model for each unit. A very popular acoustic model is the HMM, since it not only models the likelihood of a new observation vector, but also the sequentiality of the observations.

Usually, observation likelihoods are generated by the GMM belonging to each HMM state $j$. For an observation vector $o_{t}$, the observation likelihood $b_{j}$ of a GMM is calculated as shown in equation 1 .

$$
b_{j}\left(o_{t}\right)=\sum_{m=1}^{M} c_{j m} N\left(o_{t} ; \mu_{j m}, \Sigma_{j m}\right)
$$

where $M$ is the number of mixture components, $c_{j m}$ is the weight of the $m^{t h}$ component and $N(\cdot ; \mu ; \Sigma)$ is a multivariate Gaussian with mean vector $\mu$ and covariance matrix $\Sigma$.

In this work, the observation likelihoods have been obtained from the silence HMM trained using the Basque Speecon-like database [13], specifically the close-talk channel.

\subsection{The acoustic model for silence}

The HMM topology chosen for silence frames has three states, left-to-right, allowing the right-end state to connect back with the left-end state. It was trained with 13 MFCCs and 13 first and 13 second order derivatives as acoustic parameters, and 32mixtures GMMs. The frame length is $25 \mathrm{~ms}$ with a shift of 10 ms.

CMVN was applied to MFCCs, computing global means and variances from each recording session. For $N$ cepstral vectors $y=\left\{y_{1}, y_{2}, \ldots, y_{N}\right\}$, their mean $\mu_{N}$ and variance $\sigma_{N}^{2}$ vectors are calculated as defined in equations 2 and 3, respectively.

$$
\begin{gathered}
\mu_{N}(i)=\frac{1}{N} \sum_{n=1}^{N} y_{n}(i) \\
\sigma_{N}^{2}(i)=\frac{1}{N} \sum_{n=1}^{N}\left(y_{n}(i)-\mu_{N}(i)\right)^{2}
\end{gathered}
$$

where $i$ is the $i^{t h}$ component of the vector.

The cepstral features are then normalized using the calculated mean and variance vectors, as given in equation 4 . Thus, each normalized feature has zero mean and unit variance.

$$
\hat{y}_{n}(i)=\frac{y_{n}(i)-\mu_{N}(i)}{\sigma_{N}(i)}
$$




\subsection{The impact of CMVN}

The use of CMVN has a significant impact on the curves that observation likelihoods form. When testing a sample signal and computing frame by frame the observation likelihoods at each state of the silence HMM, very different curves are obtained depending on weather CMVN is applied or not. Figure 1 illustrates this difference. The middle and bottom diagrams show the curves formed by the observation log-likelihoods generated by each HMM state $s_{0}, s_{1}$ and $s_{2}$, without and with normalization respectively, through a utterance composed of four words. In this case, the normalization has been performed using the means and variances computed from the file.

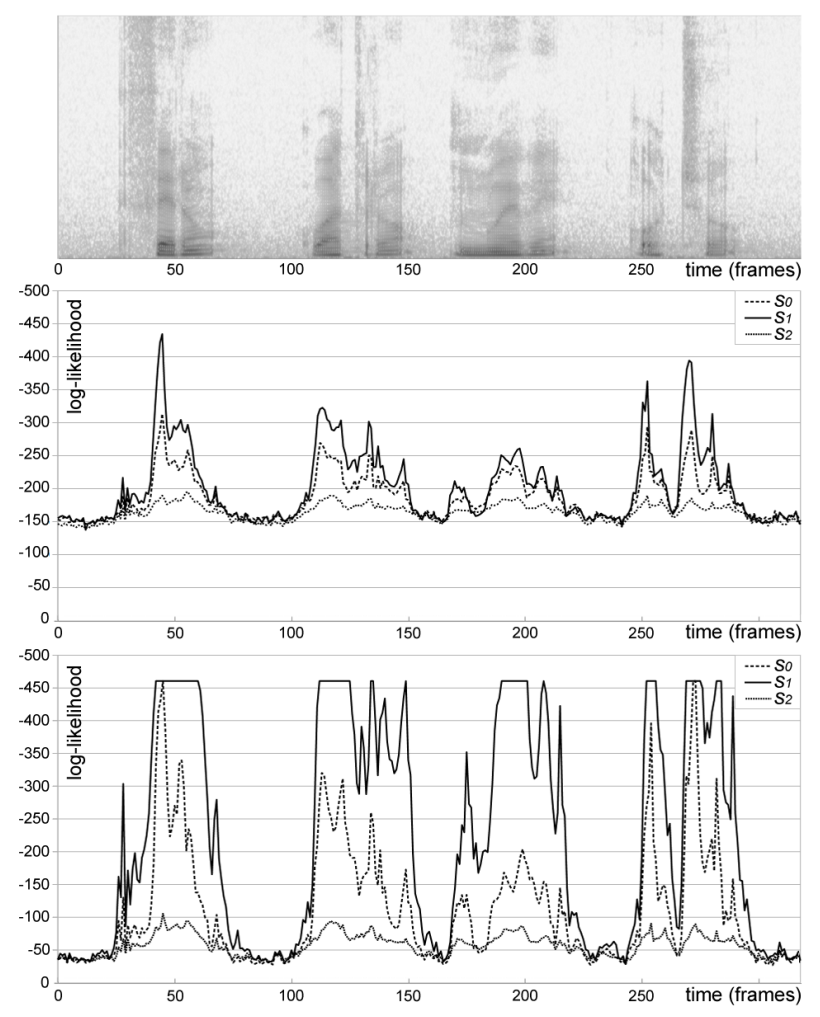

Figure 1: Spectrogram (top) and observation log-likelihoods along time (frames) generated by the left state $\left(s_{0}\right)$, central state $\left(s_{1}\right)$ and right state $\left(s_{2}\right)$ of the silence HMM without CMVN (middle) and applying CMVN (bottom).

The curves in the bottom diagram (with CMVN), compared with the ones in the middle diagram (without CMVN), look more abrupt. This fact can be used to better discern between speech and non-speech.

\subsection{The central state of the silence HMM}

In any three-state HMM, the central state is a priori the most stable state of the model, since the left and right states have to cope with transitions between models. It makes sense that the same will happen to the silence HMM, where left and right states have to model transitions between silence and speech.

Looking back at Figure 1, we can see that, indeed, the curves generated by the central state $\left(s_{1}\right)$ are, in both cases (with and without cepstral normalization), much more discriminative than the curves corresponding to the states at the ends, which are more irregular.

\subsection{Robustness against different $S N R$ values}

Another interest point to focus on in a VAD is its robustness for different recording conditions. As an example, we have chosen four signals from the Spanish SpeeCon database [14] to illustrate the impact of the recording distance on the observation likelihood curves. These four signals correspond to the same utterance, but were recorded by means of four different microphones: a headset (channel $C_{0}$ ), a lavalier (channel $C_{1}$ ), a medium-distance cardioid microphone (0.5-1 meter, channel $C_{2}$ ) and a far-distance omnidirectional microphone (channel $C_{3}$ ). Each of these channels represents a different $S N R, C_{0}$ being the cleanest (around $20 \mathrm{~dB}$ ) and $C_{3}$ the noisiest $(0 \mathrm{~dB})$.

Figure 2 shows the observation log-likelihoods generated by the central state of the silence HMM trained with the Basque Speecon-like database. The utterance is the same as the one in Figure 1 (note that the signal in Figure 1 corresponds to the $C_{1}$ signal in Figure 2). The darkest curve corresponds to the $C_{0}$ channel and the lightest one to the $C_{3}$ channel.

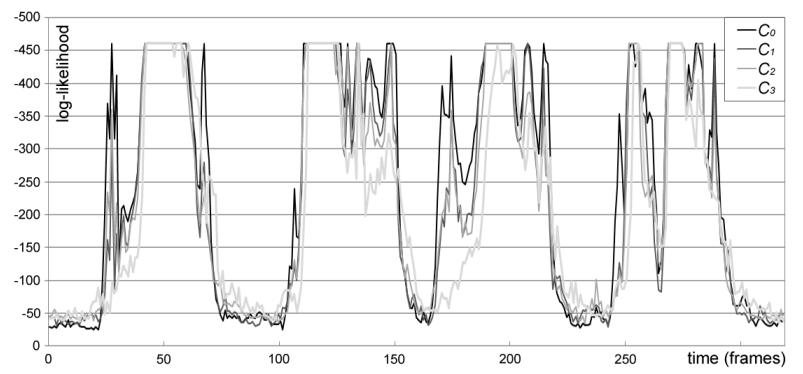

Figure 2: Observation log-likelihoods along time obtained at the central state $\left(s_{1}\right)$ of the silence HMM when processing different channels $\left(C_{0}, C_{1}, C_{2}, C_{3}\right)$.

The curves show that, as expected, a degradation occurs when the signals recorded at farther distances are processed, but even so the curves remain rather discriminative. For $C_{3}$ signals, the most adverse effect occurs at the initial and ending phones, where, depending on the phone, likelihoods can be very similar to those of the noisy silence. This happens mostly when the initial phone is a noisy phone. However, the curves show a good behaviour for $C_{1}$ and $C_{2}$, with likelihood profiles very similar to those obtained for $C_{0}$ signals.

\section{Data preparation}

To assess the stability of the observation likelihood curves generated by the central state of the silence HMM, a VAD accuracy experiment has been carried out, setting different thresholds to label frames as speech or silence.

\subsection{The databases}

Two databases have been chosen for the experiments: first, the Noisy TIMIT spech database [15], to analyse weather a threshold could be set for different $S N R$ conditions. The second database is the ECESS subset of the Spanish Speecon database [16], which has been used to test the validity of that threshold.

1. Noisy TIMIT spech database: it contains approximately 322 hours of speech from the TIMIT database [17] modified with different additive noise levels. However, we have chosen only babble and white noises, as the most natural ones. Noise levels vary in $5 \mathrm{~dB}$ steps and ranging from 50 to $5 \mathrm{~dB}$. The database contains 630 different 
speakers, with 10 utterances per speaker: 6300 files for each noise level. The total speech content in the database is $86.57 \%$ (not well balanced), and the label files are the ones belonging to the classic TIMIT database. All audio files are presented as single channel $16 \mathrm{kHz} 16-\mathrm{flac}$, but have been converted to 16-bit PCM.

2. ECESS subset of the Spanish Speecon database: it was used in the ECESS evaluation campaign of voice activity and voicing detection in 2008. It includes 1020 utterances recorded in different environments (office, entertainment, car and public place) distributed among the $C_{0}, C_{1}, C_{2}$ and $C_{3}$ subsets (total number of files: 4080). There are 60 different speakers each of which utters 17 sentences. The total speech content in the database is $55.77 \%$ (well balanced), and it contains reference speech and silence labels specifically designed to assess different VAD algorithms. The signals in the database were recorded at $16 \mathrm{kHz}$ and 16 bit per sample.

Each file's features have been normalized off-line, with the means and variances calculated from the file itself. The on-line performance has been left for future research.

\subsection{Error metrics}

The VAD accuracy experiment consists in evaluating the ability of the system to discriminate between speech and silence segments at different $S N R$ levels, in terms of silence errorrate $\left(E R_{0}\right)$ and speech error-rate $\left(E R_{1}\right)$. These two rates are computed as the fractions of the silence frames and speech frames that are incorrectly classified $\left(N_{0,1}\right.$ and $N_{1,0}$, respectively) among the number of real silence frames and speech frames in the whole database ( $N_{0}^{r e f}$ and $N_{1}^{r e f}$, respectively), as shown in equation 5 . In addition, the $T E R$ (total error rate) has also been computed as the average of the $E R_{0}$ and $E R_{1}$ (equation 6).

$$
\begin{gathered}
E R_{0}=\frac{N_{0,1}}{N_{0}^{r e f}} \times 100 ; E R_{1}=\frac{N_{1,0}}{N_{1}^{r e f}} \times 100 \\
T E R=\frac{E R_{0}+E R_{1}}{2}
\end{gathered}
$$

A minimum duration of 15 frames both for speech and silence segments was set. This value was empirically chosen after some preliminary experiments.

\section{VAD experiments}

Initially, we have analysed whether a threshold can be set for VAD purposes, considering the various $S N R$ values. Then, we have tested that threshold in a separate database, and, in addition, a validity test has been carried out comparing the results with those obtained with three standard VAD algorithms.

\subsection{Analysis of the decision threshold}

Different thresholds have been considered to label frames as speech or silence. Results are shown in Figure 3, both for babble noise (left) and white noise (right).

For the cleanest signals $(S N R=50 d B)$, the equal error rate $(E E R)$ points of $E R_{0}$ and $E R_{1}$ curves are located near -200 . However, as the $S N R$ gets lower, the $E E R$ points move towards higher values. In the case of white noise, this shift reaches the -120 value for $5 d B$.
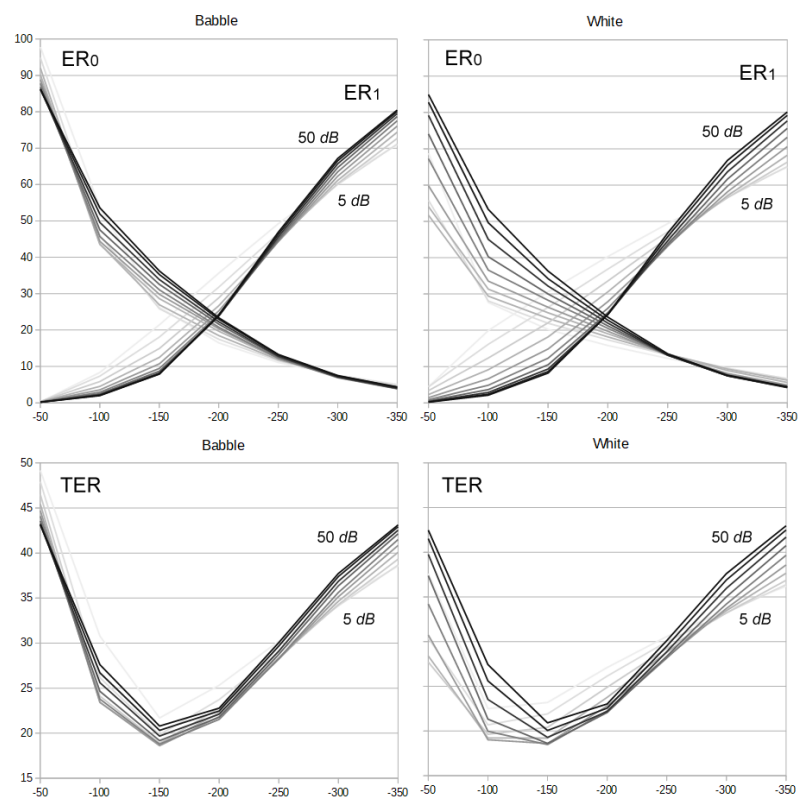

Figure 3: $E R_{0}$ and $E R_{1}$ (top) and TER (bottom) for different decision threshold values when testing the signals of $S N R 50$ to $5 \mathrm{~dB}$ in the babble noise subset (left) and the white noise subset (right) of the Noisy TIMIT database.

Regarding the error rates, the minimum $T E R$ s are obtained at $T h=-150$, except for 5,10 and $15 d B$ in white noise subset, which occur at -100 . Thus, we can consider the point of $T h=-150$ as the most valid threshold. Some $E R_{0}$ and $E R_{1}$ values obtained for $T h=-150$ are shown in Table 1 .

Table 1: $T E R, E R_{0}$ and $E R_{1}$ for $T h=-150$ on the signals of $S N R$ 50,35, 20 and $5 \mathrm{~dB}$ in the babble noise (left) and white noise (right) subsets of the Noisy TIMIT database.

\begin{tabular}{lcccccc}
\hline & \multicolumn{3}{c}{ Babble } & \multicolumn{3}{c}{ White } \\
& $E R_{0}$ & $E R_{1}$ & $T E R$ & $E R_{0}$ & $E R_{1}$ & $T E R$ \\
\hline $50 d B$ & 34.89 & 6.71 & 20.80 & 34.88 & 6.95 & 20.92 \\
$35 d B$ & 30.87 & 7.48 & 19.18 & 28.05 & 9.18 & 18.62 \\
$20 d B$ & 26.35 & 11.25 & 18.80 & 21.53 & 16.89 & 19.21 \\
$5 d B$ & 22.60 & 20.78 & 21.70 & 15.49 & 30.90 & 23.20 \\
\hline
\end{tabular}

For $T h=-150$, the minimum $E R_{1}$ is 6.71 , at $50 \mathrm{~dB}$. As expected, the $E R_{1}$ increases as the $S N R$ decreases. However, notice that the $T E R$ does not present the minimum at $50 \mathrm{~dB}$, neither in the babble noise subset nor in the white noise subset, as might be expected.

\subsection{Testing}

The threshold calculated in the previous section has been applied to the files of ECESS subset of the Spanish Speecon database. 4080 files have been tested (1020 in each $C_{i}$ subset). Results are shown in Table 2.

The results obtained for the ECESS subset using the threshold calculated from the Noisy TIMIT are very good. Compared with the best result obtained for the Noisy TIMIT (see $50 \mathrm{~dB}$ row in Table 1), much lower $E R_{0}$ and $E R_{1}$ have been obtained. The error rates, as expected, increase as $S N R$ decreases, al- 
Table 2: TER, ER $R_{0}$ and $E R_{1}$ with $T h=-150$ on the signals of channels $C_{0}, C_{1}, C_{2}$ and $C_{3}$ in the Spanish Speecon database.

\begin{tabular}{llll}
\hline & $E R_{0}$ & $E R_{1}$ & $T E R$ \\
\hline$C_{0}$ & 6.21 & 2.74 & 4.48 \\
$C_{1}$ & 4.22 & 6.13 & 5.18 \\
$C_{2}$ & 7.10 & 6.00 & 6.55 \\
$C_{3}$ & 9.46 & 6.45 & 7.96 \\
\hline
\end{tabular}

though the best silence error rate is obtained for the $C_{1}$ channel.

Additionally, a tuning has been performed for $E R_{1}$ reduction. Indeed, for speech processing, it is important to reduce the $E R_{1}$ as much as possible, so that the minimum number of speech frames are lost for the next stage. For that purpose, we have sought to reduce the impact of non-speech to speech boundaries, setting an additional margin of 5 and 10 frames around the speech segments. Results are shown in Table 3.

Table 3: $T E R, E R_{0}$ and $E R_{1}$ for 5 and 10 frames long speechsegment margins, with $T h=-150$ for the signals of channels $C_{0}, C_{1}, C_{2}$ and $C_{3}$ in the ECESS subset of the Spanish Speecon database.

\begin{tabular}{ccccccc}
\hline & \multicolumn{3}{c}{5 frames } & \multicolumn{3}{c}{10 frames } \\
& $E R_{0}$ & $E R_{1}$ & $T E R$ & $E R_{0}$ & $E R_{1}$ & $T E R$ \\
\hline$C_{0}$ & 10.84 & 1.29 & 6.07 & 15.68 & 0.79 & 8.24 \\
$C_{1}$ & 7.94 & 3.47 & 5.71 & 12.42 & 2.30 & 7.36 \\
$C_{2}$ & 10.91 & 3.50 & 7.21 & 15.39 & 2.47 & 8.93 \\
$C_{3}$ & 13.29 & 3.95 & 8.62 & 17.59 & 2.89 & 10.24 \\
\hline
\end{tabular}

The table shows that $E R_{1}$ reduces and $E R_{0}$ increases. $T E R$ increases as well, because $E R_{0}$ increases faster than $E R_{1}$ reduces. All in all, the use of a margin around speech segments allows decreasing significantly $E R_{1}$, with a not very significant resulting $T E R$ degradation.

\subsection{Comparison with other systems}

In order to validate the previous results, our results have been compared with the outcomes of three popular standard VAD algorithms carried out in a previous work [18]. These systems are standard defined by ITU (International Telecommunication Union) and ETSI (European Telecommunications Standards Institute):

1. The VAD algorithm of the ITU G.729 system [19].

2. The AFE-FD (frame-dropping mechanism) algorithm implemented in ETSI AFE-DSR (Advanced Front-End for Distributed Speech Recognition) [20].

3. The AFE-NR (noise reduction system) algorithm implemented in ETSI AFE-DSR [20].

Table 4 shows the results obtained for the three VAD systems along with the proposed method (using $T h=-150$ and a margin of 10 frames), over the same dataset (4080 files from the ECCESS subset). Regarding $E R_{1}$, the AFE-FD gets better results, and also the AFE-NR for $C_{0}$ and $C_{1}$. However both systems show the disadvantage of getting very high $E R_{0}$ for all the channels (the lowest value is $38.10 \%$ ). This means that many silence frames will be sent to the recognizer. The $E R_{0}$ in our results are between 12.42 and $17.59 \%$.
Table 4: Comparison of different VAD algorithm results at four SNR levels

(a) Silence error rates $\left(E R_{0}\right)$

\begin{tabular}{ccccc}
\hline & G.729 & AFE-FD & AFE-NR & Prop. \\
\hline$C_{0}$ & 56.06 & 63.88 & 58.23 & 15.68 \\
$C_{1}$ & 70.23 & 54.75 & 55.96 & 12.42 \\
$C_{2}$ & 59.54 & 52.10 & 38.10 & 15.39 \\
$C_{3}$ & 70.49 & 50.10 & 47.65 & 17.59 \\
\hline
\end{tabular}

(b) Speech error rates $\left(E R_{1}\right)$

\begin{tabular}{ccccc}
\hline & G.729 & AFE-FD & AFE-NR & Prop. \\
\hline$C_{0}$ & 3.63 & 0.03 & 0.62 & 0.79 \\
$C_{1}$ & 9.28 & 0.23 & 1.98 & 2.30 \\
$C_{2}$ & 18.19 & 0.48 & 4.83 & 2.47 \\
$C_{3}$ & 17.22 & 1.41 & 8.30 & 2.89 \\
\hline
\end{tabular}

\section{Conclusions}

In this paper, we have assessed the usefulness of the observation likelihood generated by the central state GMM of a silence HMM trained using CMVN, as a possible basis on which to build a VAD system. We have seen that a good classification between speech and silence can be performed, just by setting a threshold in the curves that observation likelihoods form.

The silence HMM has been trained using the close-talk channel from the Basque Speecon-like database. Then, a threshold analysis has been carried out, processing the babble and white noise files of the Noisy TIMIT database. As a conclusion, we have noticed that the minimums error rates occur at the same likelihood point in $17 S N R$ values out of a total of 20. This point is the one we have chosen as the threshold.

This threshold has been tested with a separate database: the ECESS subset of the Spanish Speecon database. The results obtained for this database are even better than those obtained for the Noisy TIMIT, which leads us to think that the silence observation likelihood behaves similarly on different channels.

Additionally, the results of the test have been compared with three different standard VAD systems. Although the best speech error rates have not been achieved with the use of the decision threshold, we have got the best silence error rates. Our results are quite competitive; actually, the best total classification rates have been obtained.

As a final conclusion, competitive results are obtained just by setting a decision threshold to the silence observation likelihood curves. This fact has been applied in [21], where a method called Multi-Normalization Scoring (MNS) is used to explode the discriminative potential of the observation likelihood scores. Robust on-line results are shown in that paper, where the scores obtained with MNS are classified with a Multi-Layer Perceptron (MLP). This issue and others related to the selection of the optimal threshold are being investigated currently in our laboratory.

\section{Acknowledgements}

This work has been partially supported by the EU (FEDER) under grant TEC2015-67163-C2-1-R (RESTORE) (MINECO/FEDER, UE) and by the Basque Government under grant KK-2017/00043 (BerbaOla). 


\section{References}

[1] M. K. Mustafa, T. Allen, and K. Appiah, Research and Development in Intelligent Systems XXXI. Springer International Publishing, 2014, ch. A Review of Voice Activity Detection Techniques for On-Device Isolated Digit Recognition on Mobile Devices, pp. 317-329.

[2] T. Virtanen, R. Singh, and B. Raj, Techniques for Noise Robustness in Automatic Speech Recognition, 1st ed. Wiley Publishing, 2012.

[3] S. G. Tanyer and H. Ozer, "Voice activity detection in nonstationary noise," IEEE Transactions on Speech and Audio Processing, vol. 8, no. 4, pp. 478-482, 2000.

[4] J. Tatarinov and P. Pollák, "Hmm and ehmm based voice activity detectors and design of testing platform for vad classification," Digital Technologies, vol. 1, pp. 1-4, 2008.

[5] H. Veisi and H. Sameti, "Hidden-Markov-model-based voice activity detector with high speech detection rate for speech enhancement," vol. 6, no. 1, pp. 54-63, 2012.

[6] Ó. Varela, R. S. Segundo, and L. A. Hernández, "Combining pulse-based features for rejecting far-field speech in a HMMbased Voice Activity Detector," vol. 37, no. 4, pp. 589-600, 2011.

[7] D. Enqing, L. Guizhong, Z. Yatong, and C. Yu, "Voice activity detection based on short-time energy and noise spectrum adaptation," in ICSP 2002 - $6^{\text {th }}$ International Conference on Signal Processing Proceedings, August 26-30, Beijing, China, Proceedings. IEEE, 2002, p. 464467

[8] Y. W. Tan, W. J. Liu, W. Jiang, and H. Zheng, "Hybrid svm/hmm architectures for statistical model-based voice activity detection,' 2014, pp. 2875-2878.

[9] T. Hughes and K. Mierle, "Recurrent neural networks for voice activity detection," 2013, pp. 7378-7382.

[10] S. Thomas, S. Ganapathy, G. Saon, and H. Soltau, "Analyzing convolutional neural networks for speech activity detection in mismatched acoustic conditions," 2014, pp. 2519-2523.

[11] Y. Obuchi, "Framewise speech-nonspeech classification by neural networks for voice activity detection with statistical noise suppression," in ICASSP, 2016, pp. 5715-5719.

[12] M. Westphal, "The use of cepstral means in conversational speech recognition," in EUROSPEECH 1997 - $5^{\text {th }}$ European Confer ence on Speech Communication and Technology, September 22 25, Rhodes, Greece, Proceedings. ISCA, 1997, pp. 1143-1146.

[13] I. Odriozola, I. Hernaez, M. I. Torres, L. J. Rodriguez-Fuentes, M. Penagarikano, and E. Navas, "Basque speecon-like and Basque speechdat MDB-600: speech databases for the development of ASR technology for Basque," in LREC 2014, Ninth International Conference on Language Resources and Evaluation, May 26-31, Reykjavik, Iceland, Proceedings, 2014, pp. 26582665 .

[14] D. Iskra, B. Grosskopf, K. Marasek, H. van den, F. Diehl, and A. Kiessling "Speecon speech databases for consumer devices: Database specification and validation," in LREC 2002, Third International Conference on Language Resources and Evaluation May 27-31, Las Palmas, Spain, Proceedings, 2002, pp. 329-333.

[15] A. Abdulaziz and V. Kepuska, "Noisy timit speech (ldc2017s04)," 3 2017. [Online]. Available: http://hdl.handle.net/11272/UFA9N

[16] B. Kotnik, P. Sendorek, S. Astrov, T. Koç, T. Çiloglu, L. D Fernández, E. R. Banga, H. Höge, and Z. Kacic, "Evaluation of voice activity and voicing detection," in INTERSPEECH 2008 $8^{\text {th }}$ Annual Conference of the International Speech Communication Association, September 22-26, Brisbane, Australia, Proceedings, 2008, pp. 1642-1645.

[17] J. Garofolo, L. Lamel, W. Fisher, J. Fiscus, and D. Pallett, "Darpa timit acoustic-phonetic continous speech corpus cd-rom. nist speech disc 1-1.1," NASA STI/Recon Technical Report N, vol. 93,1993
[18] I. Luengo, E. Navas, I. Odriozola, I. Saratxaga, I. Hernaez, I. Sainz, and D. Erro, "Modified LTSE-VAD algorithm for applications requiring reduced silence frame misclassification." in LREC 2010, Seventh International Conference on Language Resources and Evaluation, May 17-23, Valletta, Malta, Proceedings, 2010, pp. 1539-1544.

[19] P. Setiawan, S. Schandl, H. Taddei, H. Wan, J. Dai, L. B. Zhang, D. Zhang, J. Zhang, and E. Shlomot, "On the itu-t g.729.1 silence compression scheme." in EUSIPCO 2008 - $16^{\text {th }}$ European Signal Processing Conference, August 25-28, Lausanne, Switzerland, Proceedings, 2008, pp. 1-5.

[20] E. Standards, "Speech processing, transmission and quality aspects (stq); distributed speech recognition; front-end feature extraction algorithm; compression algorithms," ETSI Standards, European Telecommunications Standards Institute, vol. ES 201108 Recommendation, 2002

[21] I. Odriozola, I. Hernaez, and E. Navas, "An on-line VAD based on Multi-Normalisation Scoring (MNS) of observation likelihoods," Expert Systems with Applications (ESwA), vol. 110, pp. 52-61, 2018 . 